\title{
Using Porous Media to Enhancement of Heat Transfer in Heat Exchangers
}

\author{
Sonia Rashidian ${ }^{1}$, Mohamad Reza Tavakoli ${ }^{* 2}$ \\ ${ }^{1}$ Department of Mechanical Engineering, Isfahan University of Technology, Isfahan, 84156-83111, Iran \\ Email: sonia.rashidian@gmail.com \\ ${ }^{2}$ Department of Mechanical Engineering, Isfahan University of Technology, Isfahan, 84156-83111, Iran \\ Email: mrtavak@cc.iut.ac.ir
}

\begin{abstract}
According to increasing human needs for energy and to avoid energy waste, researchers are struggling to increase the efficiency of energy production and energy conversion. One of these methods is increasing heat transfer and reducing heat dissipation in heat exchangers. Using porous materials in the fluid flow is one of the passive methods to increase heat transfer in heat exchangers. The existence of porous media in the flow path, improve the matrix of thermal conductivity and effective flow thermal capacity and also matrix of poroussolid increase radiation heat transfer, especially in two phase flow (gas-water) systems. In this paper, recent studies on the effect of using porous media on enhancement the amount of heat transfer in heat exchangers has been investigated via using porous media with difference porosity percentage, material and geometric structure in the flow path in numerical simulations and laboratory studies.
\end{abstract}

Keywords-Porous media, Heat transfer enhancement, Heat exchanger.

\section{INTRODUCTION}

Energy consumption is one of the most important issues that man has faced over the past decades. Providing clean and environmentally friendly energy is of great importance to developed countries. Among the various types of energy used today, more than $70 \%$ of it is exchanged as heat energy. In many industrial systems and processes, the heat must be given as input to the system or, ultimately, the energy must be exhaust from the system. By increasing the need for energy in the world, strengthening the process of heat transfer and reducing energy losses is of great importance [1].

Better design of heat exchangers and their wider application to retrieval of the far-off heat industry can have a significant effect on the preservation of fuel resources and the prevention of environmental pollution. The key to increasing heat transfer is to reduce thermal resistance. This results in smaller heat transfer systems with lower cost and better efficiency. Heat transfer and control are a very important issue in high-flux systems, including reactors and nuclear reactors, microelectronic systems and micro-chemical reactors, and many other applications. Today, porous materials are used in many industrial applications to control the heat transfer and insulating of the systems. The most important application of porous materials is the insulating of furnaces and boilers and the transfer of energy in the geothermal and oil industries.

\section{HEAT TRANSFER ENHANCEMENT METHODS}

In recent decades, many studies have been conducted to enhance heat transfer, focusing on finding methods that, in addition to increasing heat transfer, have more efficiency. Such methods lead to lower energy consumption and less costly and less expensive equipment, with higher thermal efficiency [2]. As increasing efficiency and improving energy consumption in the industry have always been a concern of the researchers, improving heat transfer in heating and cooling systems is no exception. Therefore, extensive research to enhance the transmission methods Heat is used to reduce heat dissipation in these systems. Bergles, In his book [3], introduced fourteen ways to increase heat transfer in heat exchangers. These methods can be divided into two active and passive categories. Passive methods do not require any external power source to increase heat transfer. But active methods require an external power source. Table 1 presents examples of these two methods. Passive methods are preferable to active methods because of simple production, low cost, long life and convenient use [4]. One of the non-active methods that has been considered in recent decades to increase heat transfer in heat exchangers is the use of porous media. Porous media play an important role in the industry due to their unique properties. In the following, the porous environment and its application are discussed. 


\begin{tabular}{|c|c|}
\hline Passive methods & Active methods \\
\hline Surface coating & Mechanical method \\
\hline Rough surfaces & Vibrate surface \\
\hline Extended Surfaces & Fluid Vibration \\
\hline Removable devices & Electrostatic fields \\
\hline Surface tension devices & Injection \\
\hline Additives for liquids & Suction \\
\hline Rotational flow devices & Spraying devices \\
\hline
\end{tabular}

\section{POROUS MEDIUM}

When discussing the porous medium, it means a material that is composed of a network of solids and empty spaces, with the assumption that the solid network is completely rigid. The existence of this network is indispensable for porous media. This means that the solid is not a porous medium without a network of empty spaces. Empty spaces between solids allow fluid to move in porous matter. The fluid path inside the cavities is very complex, at small and comparable porosity measurements, flow quantities such as speed and pressure will be completely irregular, therefore, a study on a microscopic scale is not possible. Therefore, to analyze the porous medium, the continuous environment is used. Figure 1, shows the examples of natural porous material. Examining empirical results has shown that in areas with a large number of pores, the average of the behavioral flow rates with the base are relative to time and space, and theoretical discussions could be devised for them. It may depend on the porosity of the porous medium to be considered as an observer. If the observer's distance is small, the observer will see only one or two ducts; then, in the traditional methods of fluid mechanics, fluid behavior can be described. But if the environmental distance is high, there will be plenty of ducts and holes in the observer's eyes; then, volumetric modalities will be much more useful and efficient for describing fluid behavior and simplifying the problem. [5].

The expression unit is the ppi number of porous medium cavities. The larger the number, the more caverns and the smaller the cavities are smaller [6]. Most porous media are rigid and rigid, but can sometimes be slightly deformed. In continuous spaces, one or more streams flow. In single-phase mode, empty spaces are saturated only by a fluid, while in two-phase flows; liquid and gas saturate empty spaces. The nature of porosity and nonrepetitive algorithms of these environments have many complications for the analysis of these environments. The fluid flows in porous layers and causes the transfer of mass and energy between these materials. The study of heat transfer in non-homogeneous porous media is not easy [7]; it is also important to analyze and design various catalysts and thermal insulators.

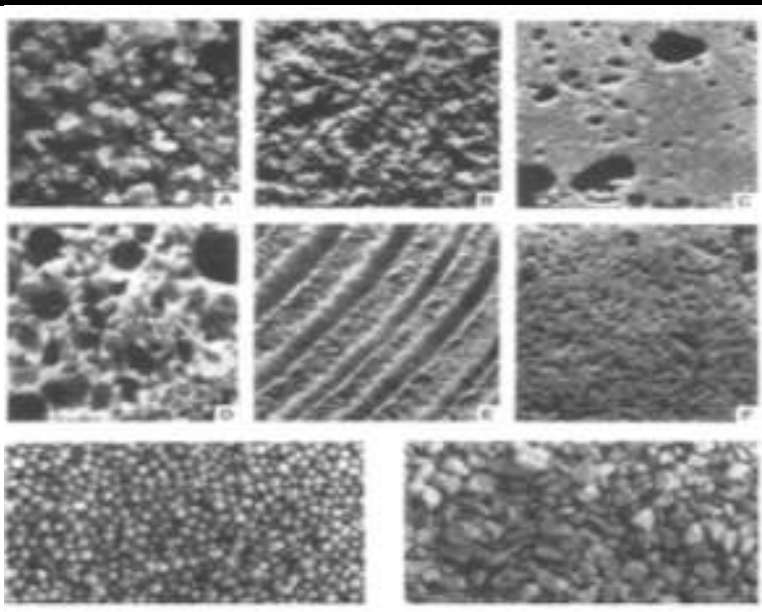

Fig. 1: Examples of natural porous material: (A) beach sand, $(B)$ sandstone, $(C)$ limestone, $(D)$ rye bread, $(E)$ wood, $(F)$ human lung. Bottom: Granular porous materials used in the construction industry, 0.5-cm-diameter Liapor spheres (left), and 1-cm-size crushed limestone (right) [5]

\section{POROUS MEDIUM CHARACTERISTICS}

In this section, the parameters that are used to identify the porous medium are introduced.

\subsection{Porosity}

The porosity $(\varphi)$ of a material is the amount of storage capacity that can hold the fluid, in other words, it can be said to be the porosity of the blank volume ratio to the total volume. In another definition, the concept of effective porosity is used, which is the ratio of empty spaces interconnected to total volume [8]. In natural environments, porosity does not exceed $0.6 \%$. In a bed of matched spheroid solids, porosity can vary from 0.2595 to 0.4644. If the particles do not match, porosity will be less. For some porous human materials such as metal foams, porosity is close to 1 . If in the porous medium the cavities flow and the fluid contained within these cavities completely fill them, so to say, this porous medium is said to be a saturated porous medium. An unsaturated porous medium refers to an environment in which the fluid, in general, only fills part of the space in the cavities or does not have all the cavities, so the fluid cannot be in the entire cavity [9].

\subsection{Infiltration Coefficient}

The characteristic of other porous media is the permeability of $\eta$, which is the ability of the material to pass fluid through itself. The unit of this coefficient is equal to the length to the power of two $\left(\mathrm{L}^{2}\right)$. This characteristic has been used in the relationship between the velocity and the pressure drop in a porous medium as part of the proportional constant. The form of this relation, known as the Darcy's law [10], is: 


$$
u=-\frac{k}{\mu} \frac{\partial P}{\partial x}
$$

where $\mathrm{u}$ and $\mathrm{p}$ are the velocity and pressure drop in a porous medium respectively. $\mathrm{k}$ is independent of the flow specification and depends on the geometry of the porous medium. The porous medium with permeability 1 allows the flow of fluid to pass through the viscosity 1 Pa.s at a speed of $1 \mathrm{~m} / \mathrm{s}$ and with a pressure difference of $1 \mathrm{~Pa}$ in a cross section of $1 \mathrm{~m}^{2}$. This coefficient is called the specific permeability or intrinsic permeability of the porous medium. Researchers working in the field of geophysics often use a common penetration unit called Darcy, which is $987 \times 1017$ square meters. The porosity of porous materials in nature is very diverse. For example, the penetration coefficient of clay is lower than coal, and coal is less clean sand. The permeability coefficient of the clean gravel is higher than all of them [10]. The prediction of porosity coefficient of permeability is one of the issues that many scientists and researchers deal with. These studies are particularly complicated, especially for porous materials, with the internal structure of the pores irregular and unobtrusive. The researchers concluded that the infiltration coefficient was influenced by parameters such as the porous media bed structure, porosity coefficient, particle geometry of the substrate, homogeneity or non-homogeneity of the surface. Many theoretical and empirical studies have been carried out on a bed of spherical particles or fibers of various materials have been used to model porous media. The use of these artificial porous media, due to the network structure and repeating pattern, creates homogeneous bedding, making it easier to check the porous environment and make general rules simpler $[11,12]$.

\section{APPLICATION OF POROUS MEDIUM}

The porous environment and the study of the phenomenon of heat transfer and flow in it, has a special place in technology and industry. Although studies on porous media have attracted scientists and researchers from recent decades, the importance of this has led researchers of recent years to consider parameters that affect heat transfer in porous media. Various applications have been made of porous media applications such as heat exchangers, porous blades, porous burners, solar collectors with porous adsorbents and many other cases [13].

\section{THE ROLE OF POROUS MEDIA IN INCREASING HEAT TRANSFER}

Porous media have a large contact surface with fluids, which can enhance the heat transfer effect. The porous medium not only changes the flow field conditions and causes the frontal layer to thinner, but also the conduction heat transfer coefficient is usually higher than that of the fluid studied. As a result, the introduction of a porous medium into a fluid channel effectively improves the thermal transfer properties [14]. Also, for open cellular porous media, the presence of multiple paths that are intense heat conductors can increase heat transfer; another reason is the increase in heat transfer, the flow of the porous matrix and its high mixing. Applying the porous medium, depending on the permeability of the environment, forces the fluid to escape from the central region to the outer regions, which reduces the thickness of the boundary layer and increases the heat transfer rate. The porous medium also corrects effective thermal conductivity and effective heat capacity and fluid heat capacity, and, in a system that has a gas flow, the solid matrix also enhances the heat transfer rate. Heat transfer reinforcement occurs through three mechanisms: redistribution of current, thermal conduction modification, and correction of environmental radiation properties [15].

The study of the hydraulic properties of porous media is important because the transfer of heat can be considerably increased by adding a porous medium to a heat exchanger. But, on the other hand, the fluid passes through the porous matrix, with greatly reduced pressure. In order to achieve the maximum heat transfer in a heat exchanger, the porous medium parameters must be optimized during the design stage, due to the fluid pressure drop. Improvement of heat transfer in thermal systems (such as heat exchangers) using a porous medium results in a significant increase in the loss of fluid pressure and fluid pumping power. Therefore, a parameter is needed for simultaneous evaluation of thermal performance and pressure drop in these systems. Accordingly, Webb and Eckert presented a parameter called the Performance Evaluation Criteria (PEC), which takes into account the simultaneous effects of heat transfer and pressure drop and is defined in equation (2) in the heat exchanger [16.17].

$P E C=\frac{N u / N u_{s}}{\left(f / f_{s}\right)^{1 / 3}}$

where, $\mathrm{Nu}$ and $\mathrm{f}$ are the Nusselt numbers and the tube pressure drop coefficient in the presence of porous material, and $\mathrm{Nu}_{\mathrm{s}}$ and $\mathrm{f}_{\mathrm{s}}$ are the Nusselt number and the coefficient of pressure drop in the simple tube. Today, cellular microstructures are widely used in heat dissipation technologies, shock absorbers and compressed heat exchangers. Thermal properties such as high heat transfer rates allow the material to be cooled or put into a 
high performance heat exchanger.

\section{RESEARCH ON THE EFFECTS OF POROUS MEDIUM}

The first studies on porous media began with Darcy studies on the soil environment and found a relationship between the fluctuations of fluid pressure flowing in it. In 1856, the Darcy introduced a legal environment for the flow of water through a porous medium as follows [18].

$$
Q=-k \cdot A \cdot \frac{d H}{d L}
$$

Another of the first studies on porous media was carried out by the Ergum, in 1952, in his study; he presented a relation to calculate the pressure drop in the channel [19]. These relations are given in the set of relations (4).

$$
\begin{aligned}
& f_{p}=\frac{150}{G r_{p}}+1.75 \\
& f_{p}=\frac{\Delta p}{L} \frac{D_{p}}{\rho v_{s}^{2}}\left(\frac{\varepsilon^{3}}{1-\varepsilon}\right) \\
& G r_{p}=\frac{\rho v_{s} D_{p}}{(1-\varepsilon) \mu}
\end{aligned}
$$

In these equations, $\Delta \mathrm{p}$ is the difference in pressure in the flow bed, $L$ is the length of the bed of the current $D_{p}$, is the equivalent diameter and $\varepsilon$ is the porosity permeability coefficient. Also, heat transfer enhancement has been widely studied in various equipment such as heat exchangers. Many scientists have worked in this direction. In 1994, the Webb discussed different methods and techniques for transfer of heat in single-phase and multi-phase fluids [20]. One of the ways to increase the speed of mass and heat transfer in different systems is to use porous media. Lauriat et al. [21] reported an increase of 50 percent in Nusselt numbers during the slow flow of porous canals over porous canals. Generally, the transfer coefficient of heat transfer is higher in systems consisting of porous media. One of the important reasons for this is the increase in the thermal conductivity of the porous matrix in different papers and papers, compared with the thermal conductivity of the fluids.

\section{NUMERICAL STUDIES}

In the field of flow modeling in porous media, a relatively large number of numerical and analytical works has been done. Examples include the following.

Mohamad [22] studies the numerical flow and heat transfer in a semi-porous tube. This research has been investigated for a completely filled pipe with porous material and a tube in which the porous material has been embedded in the tube center. In this research, the Nusselt number, the developed pressure gradient and the flow velocity profile have been calculated. In calculating the momentum and energy equations, simplifications are assumed with the assumption of a slow, two-dimensional, incommensurable, stable, thermodynamic equilibrium between porous matter and its fluid and insignificance of energy dissipation in the energy equation. In this work, the temperature of the pipe surface was assumed to be constant, the effect of increasing and decreasing the relative diameter of the porous material inside the tube, and the effect of the variation of the Darcy number on the pressure drop and Nusselt number were investigated. In figure 2, the geometry of Mohamad's problem is shown.

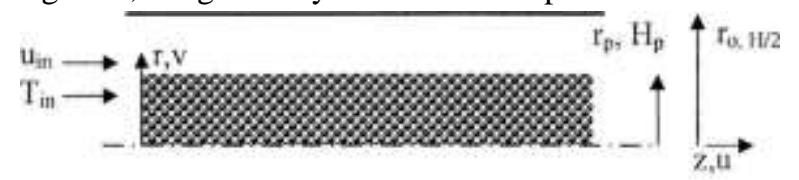

Fig. 2: The schematic of the pipe and plate, investigated by Mohamad [22]

Solving the flow and energy equations in this work is an integrated that simultaneously involves both environments. In the fluid environment without porous material its equations and in the porous medium are also solved for its equations, and for the boundary grains that are at the interface between the two environments and half of it inside the fluid and the other half The porous medium, the average harmonic value of fluid properties such as viscosity and conduction heat transfer coefficient are placed in equations. In this study, Mohamad obtained some results, including the fact that the inertial coefficient has an important effect on the Nusselt number, while the dependence of this coefficient on porous media with high permeability is not accurate. The effect of adding the inertial coefficient to the Navier-Stokes equations is to calculate the local drag force. The extended channel length is not a function of the Darcy number. The increase in the diameter of the porous material causes an increase in the pressure drop, but the Nusselt number first increases with increasing porosity of the porous material and, after reaching a certain value, is reduced again. However, the pressure drop is higher than that in which the tubes are empty and without a porous medium. For this reason, an optimal amount of porous material diameter should be found. Siavashi et al. [23] investigated the heat transfer in a tube containing aluminum metal foam with nanoparticles in the fluid. The operating fluid in this study is chosen with particles $\mathrm{Al}_{2} \mathrm{O}_{3}$, which are nanoparticles of nanoparticles. This research has been done in two modes. In the first case, the geometry is such that the porous material as well as the thermal flux is drawn from the inner wall to the tube. In the second case, 
the porous material and thermal flux are in the vicinity of the outer wall. In this study, the increase in entropy and nano-velocity effect on porous matrix has been investigated. The corresponding diagrams are plotted for the Nusselt number and the pressure over the channel. This research has been studied by assuming a slow flow for Reynolds numbers between 100 and 2000. Also, with the increase of the Darcy number of the profile, the speed is increased more rapidly. On the other hand, by increasing the porosity of the porous material, the nanofluid flows more easily and this reduces the thickness of the thermal boundary layer. In this study, the performance improvement, i.e., the ratio of heat transfer variations to pressure drop variations is shown by equation (5).

$$
P N=\frac{N u / N u_{s}}{\Delta p / \Delta p_{s}}
$$

where, $\mathrm{Nu}$ and $\Delta \mathrm{p}$ represent the Nusselt number and the pressure drop in the presence of porous matter, respectively. Similarly $\mathrm{Nu}_{\mathrm{s}}$ and $\Delta \mathrm{p}_{\mathrm{s}}$ represent the Nusselt number and the pressure drop in the internal flow without porous material. Siavashi has used two models of the Darcy Brinkman Forchimer and two-phase combination models. According to the results of this study, also taking into account the second law of thermodynamics, an optimum thickness for porous material has been proposed to reduce the entropy production rate. Shokouhmand and colleagues [24] examined the effect of porous matter on increasing the heat transfer of the channel, part of which has a porous medium. They were found to be in good agreement with the analytical solutions by placing the porous material at the side of the walls and in the core of the channel. By examining various parameters on the channel's thermal performance, such as Darcy number, porous material thickness and permeability, it was determined that the porous material position has a great effect on the thermal performance of the channel. Nazari et al. [25] investigated the heat transfer conductivity between two parallel plates containing different porous layers in an analytical study. They considered the boundary conditions in the upper and lower plates in the form of constant temperature with respect to the production of internal heat under thermal imbalance conditions. First, the heat transfer equation was extracted in each layer for solid and fluid phases, and then, considering the thermal boundary conditions in each layer, the heat transfer equations were compared to the analytical solution. The proposed analytical solution is general and valid for any number of porous layers of different thicknesses. The results of this study show the effects of the porosity ratios of each layer, the fluid and solid conductivity coefficients, and the displacement heat transfer coefficient on the cavity scale on the temperature distribution and temperature difference between the two fluid and solid phases. The results show that by increasing the ratio of solid and fluid thermal conductivity coefficients, the temperature difference between the two phase's increases. Increasing the transfer heat transfer coefficient causes the temperature rise of both solid and fluid phases. In the case where the porosity ratio of the two layers is different, at the joint location, the temperature distribution of each of the two phases is fractured. Changes in the porosity ratio in the layers can cause the maximum temperature of the two phases to be transferred from the center of the channel. The maximum temperature difference is directly proportional to the coefficient k. Maerefat et al. [26] investigated the heat transfer inside a dual-heat exchanger of metal porous material. The flow of fluid within the tubes is a turbulent heat exchanger, which is more in line with the actual operating conditions of these converters in the industry. Fluid flow and heat transfer equations have been decomposed using a finite volume method and using the Simplil algorithm on a localized network. By writing a numerical program in the Fortran language, the effect of the porous material specification and the Reynolds number of the fluid flow on heat transfer has been investigated. Figure 3 shows the geometry in this numerical study.

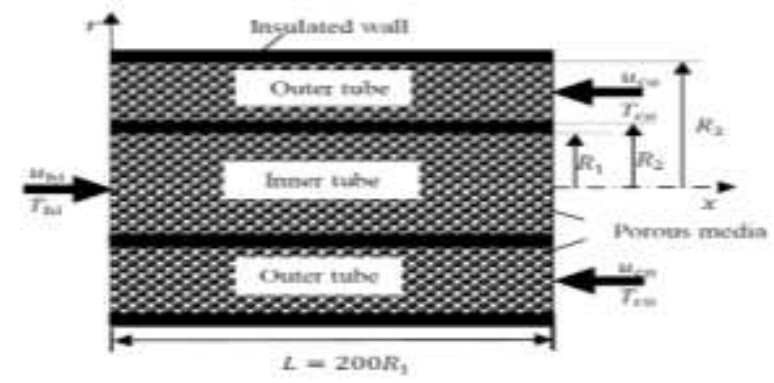

Fig. 3: Geometry investigated in Jamarani research [26]

The results of this study show that the use of porous material in the heat exchanger increases the total heat transfer coefficient of porous material and, in the best case, results in an improvement of about 7 times. Reducing porosity in the range of $0.95-0.8$ increases the porosity of the porous material and the thermal conductivity coefficient, thus improving the heat transfer in the heat exchanger, although a reduction in porosity results in a significant drop in pressure. Investigating the changes in the diameter of the porous material cavity in the range of 1 to $6 \mathrm{~mm}$ shows that increasing the diameter of the cavity increases the permeability and dimensions of the turbulent flow vortices in the porous medium, thereby increasing the turbulence of the flow and heat transfer 
And the pressure drop also decreases. They also found that heat transfer in the heat exchanger increases in the case of a turbulent fluid, in contrast to the slow flow, in the low ratios of the thermal conductivity of the porous material to the fluid. This is due to the increase in the effect of disturbances of the current, which is not present in the calm flow. As the amount of this ratio increases, the total heat transfer coefficient of the heat exchanger increases and reaches the highest value for the porous material of copper. Another result was that changing the Reynolds number of the turbulent flow from 10,000 to 80,000 does not change the heat transfer efficiency. However, the results show that the ratio of improved heat transfer in the flow is more than confluent flow. Evaluation of the performance evaluation criterion shows that with increasing the diameter of the cavity and the thermal conductivity of the porous material, the system performance can be improved, while the porosity increase of the porous material does not have much effect on this parameter. In another study, Maerefat et al. [27] examined the numerical and analytical validity of two common definitions of the Nusselt number for the transfer of heat transfer in a pipe with porous porous material.

The

first

definition

of

$N u_{1}(x)=2 R(\partial T / \partial r)_{r=R} /\left(T_{w}-T_{m}(x)\right)$ is presented

for many non-porous tubes. Different porous materials have been used in a tube.

The second definition of

$N u_{2}(x)=2 R q_{\text {Cond. }}^{\prime \prime} / h_{r e f}\left(T_{w}-T_{m}(x)\right) \quad$ is $\quad$ also

provided by various authorities for the transfer of heat in a pipe or channel with a porous medium.

The analytical results showed that for a tube without a porous material or in a pipe with a central arrangement of porous matter, the Nusselt number is equal to the two definitions, but in the boundary arrangement of the porous material in the tube, the Nusselt number obtained from the two Each other is different. In the resulting relations it was found that in the first definition of the Nusselt number, the thermal value transmitted through the fluid flow from the porous material to the fluid is not taken into account, and therefore the calculated Nusselt number of this definition is not correct. However, in the second definition, the thermal flux of the input from the porous material is considered to be fluid, and thus the Nusselt number is appropriate for this definition. In this study, the boundary arrangement of the porous material was simulated numerically in a tubular tube with a turbulent flow and the Nasset number was calculated according to both definitions. The results showed that, in different thicknesses of porous material, the Nasset number decreases according to the first definition. By increasing the heat transfer coefficient of the porous material, the Nusselt number decreases from the first definition, which is quite non-physical. Therefore, the first definition is not suitable for calculating the Nusselt number. The results of the second definition of Nusselt number show the increase of heat transfer in the tube with the porous porcelain boundary arrangement. This is completely compatible with the physics of the problem, and as such, the use of this definition is appropriate for calculating the Nusselt number in the boundary arrangement of the porous material.

In another study, the numerical study of the effect of aluminum-oxide nanowire on the heat transfer in a tube containing porous material was investigated with a mixed flow of fluid [28]. Thermal tubes have been studied in four different structures without porous material, filled with porous material, boundary and central makeup of porous material. The results show that the use of nanofluidis improves the thermal conductivity of the entire nanofluid and porous material in the tube section. As the porous material is located in the central arrangement, the total conductivity coefficient at the adjacent wall of the pipe shows the most improvement, and thus the heat of entering the fluid in this case has the highest increase. As the porous material is placed adjacent to the wall of the pipe, ie the border arrangement and the state of the porous material, due to the thermal conductivity of the porous material, the effect of nanoparticles on the total thermal conductivity is lower and, as a result of heat transfer Moving is a better place for improvement. Hamidi-Moghadam et al. [29] have investigated an analysis of the fully developed forced displacement flow in a cylindrical homogeneous porous channel. The obtained equations have been solved for temperature distribution in transverse directions, considering frictional heat due to viscous loss, in which the Darcy number and the Brinkman number are variables. The effect of heat induced by friction on the temperature distribution is investigated and analyzed for two heating and cooling processes. The results indicate that Darcy's effect on the distribution of fluid temperature is insignificant in the absence of the effects of the viscous loss term, which includes the effects of internal and friction, while, taking into account the effects of viscous losses, the temperature distribution is a large nationalization of the Darcy number And Brinkman number. For both the cooling and heating processes, the contribution of friction heating term along the walls is high and as it moves away from the walls toward the center of the channel to zero, while the contribution of the internal heating term to the channel center It is maximized and decreases to near zero as it approaches the walls. Therefore, in general, the effect of the viscous heat loss on the walls is exclusively frictional heating, and in the 
vicinity of them, the combination of internal heat and frictional heating, and in the center of the channel is exclusively internal heating. Therefore, it can be concluded that frictional heating production is confined to the walls, while domestic heating produces a large part of the canal, originating from the center of the canal. Superior Shokouhmand and Ejlali [30] Transient heat transfer was studied in a bundle of two-dimensional porous Finns, which is under the influence of a smooth fluid flow. The governing equation governing the fluid was considered outside the porous medium of the NavierStokes equation and within the porous medium of the Brinkman-Forchheimer equation. The fins were placed horizontally, two-dimensionally, with a constant porosity ratio, and the two-dimensional flow of fluid was transmitted through a forced displacement from this set. In this study, it is assumed that the fins are homogeneous and the fluid flow is continuous and continuous, and the thermal equilibrium is established for this set. The flow rotation function has been used to solve the momentum equations in the fluid and fin fluid conditions. In examining the results after obtaining the flow and temperature field, it was determined that porous fines are more efficient than other types of fines, and this improvement in thermal efficiency increases with increasing porosity coefficient, but increasing the heat transfer coefficient Conductivity reduces the heat transfer efficiency of porous fins compared to conventional nonporous fines. Also, increasing the flow velocity is ineffective in improving the ratio of heat transfer, because in this case, the fluid particles leave the porous medium at a higher rate, and due to the geometry of the problem, which looks like a channel, there is not enough time to exchange heat. The variation of the Nusselt number is also increased by increasing the speed of the fluid injection at the beginning of the Finn, so that the Nusselt number increases to a point where, after a while, the injection rate is zero, in which case the Nusselt number remains unchanged. However, the heat transfer behavior of the porous and solid Finns is similar to each other. Rezaie and Maghrebi [31] investigated the numerical simulation of a smooth natural transfer of heat transfer in a porous square box with two solid walls using Boltzmann's method. The porous medium is modeled on a macroscopic scale, and the Darcy-Brinkman-Forchemer model has been used to model porous media in the Prandel number equal 1. Figure 4 shows the geometry examined.

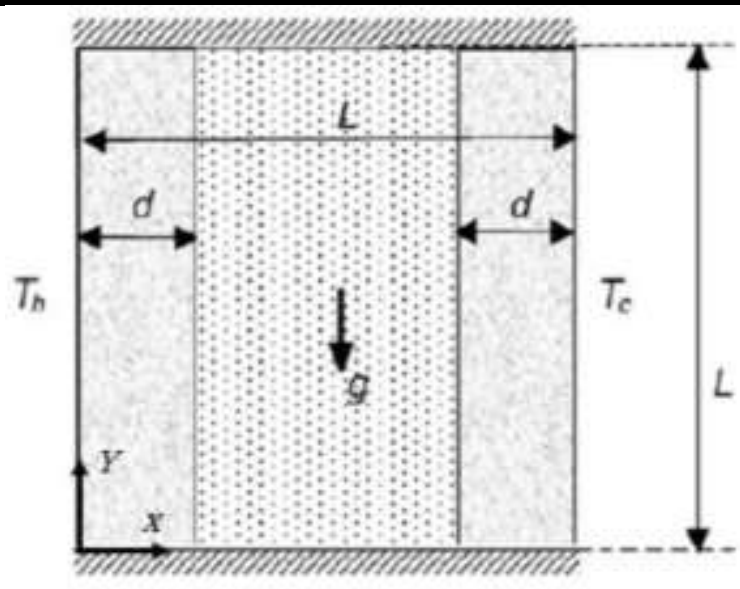

Fig. 4: Geometry studied by Rezaie and Maghrebi [31]

According to the results, it was observed that with increasing Riley number, the amount of heat transfer from the environment due to the change in the heat transfer regime from the conduction to the displacement increased, and by decreasing the Darcy number due to the decrease of the fluid permeability in the environment Porous, the amount of heat transfer decreases. By increasing the porosity coefficient due to the decrease of the resistance in the fluid path, the heat transfer rate increases in the environment, as well as by increasing the thickness of the solid walls due to the overlay of the transfer of heat transfer in the transfer The overall heat of the enclosure and the concentration of heat transfer to a thin layer in the middle of the enclosure, the amount of heat transfer from Hfzh decreases and finally, by increasing the permeability of the porous solid-wall temperature also increases the overall heat transfer from the chamber. Nazari et al. [32] examined the free heat transfer heat transfer from a vertical hot plate in a porous material. Thermal imbalance model was used to express energy equations in fluid and solid phases. The problem is analyzed for the state of the fluid source of the heat source. The equations governing the problem are extracted from the similarity method and analyzed by numerical method. The conceptual diagram of the porous medium and the coordinates of the problem are shown in figure. 5. According to the results, the increase in the suction / drop parameter reduces the thickness of the boundary layer of the temperature and leads to an increase in the local nsset number for both phases. Therefore, to achieve the thermal equilibrium between phases, the thickness of the boundary layer can be reduced. The increase in the thickness of the solid phase solid-state boundary layer will be proportional to the desire of the solid phase Nusselt number to zero. Also, in the absence of internal heat generation in the fluid phase, the Nusselt number is larger. 


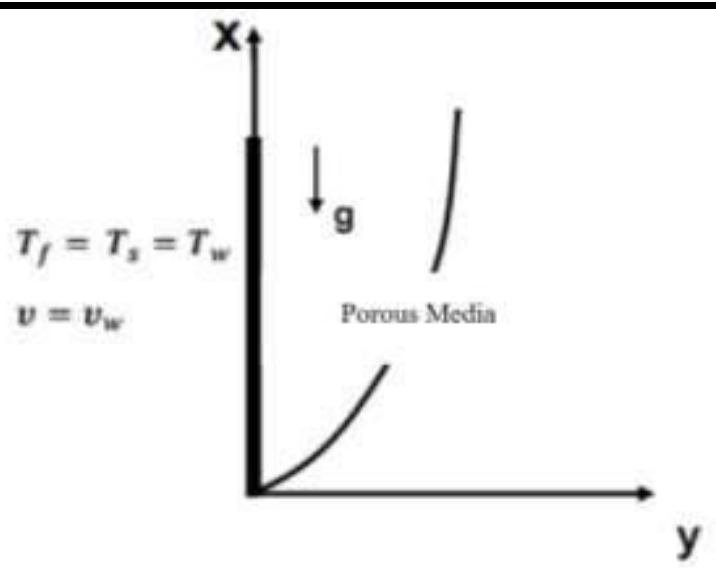

Fig. 5: Geometry examined by Nazari et al. [32]

In another study, the same people [33] have investigated numerical transfer of heat and mass transfer in porous matter in the presence of biochemical thermal flux. In this study, it is assumed that the desired environment is a homogeneous porous medium and that the Darcy model for the momentum equation is used. The porous material compartment contains two concentration components, one of the first biomass and the second component of the substance. The results show that the role of porosity in this biochemical process is significant. The porosity ratio affects the maximum temperature in the porous medium, the growth rate and consumption of the masses, and in particular the heat produced by the chemical reaction. Increasing porosity in the studied environment increases the biochemical production of heat in the compartment. Taeibi-Rahni et al. [34] A comparison and investigation of the flow and rate of forced convection heat transfer in a broadly-spaced tube were investigated numerically in a three-dimensional fashion. The porous material is partially or completely arranged in two distinct boundaries and central layers within a wider tube. The boundary condition governing the wall of the tube is constant temperature. The results show that using porous material in the central arrangement, increasing the thickness of the porous layer to about 0.75 , increases the heat transfer and then decreases. Reducing the Darcy number is also one of the factors that increase heat transfer. Of course, the increase in heat transfer is accompanied by an increase in the pressure drop, which, given the economic cost, should be considered optimally. Contrary to the central arrangement, in the border arrangement, the heat transfer is first reduced and then increased to a maximum extent when the thickness of the porous layer reaches a certain value (thickness of about $0.5)$, until it is completely porous. Kayhani and Mohebi [35] studied the forced flow and heat transfer between two parallel planes, which were partially filled with porous media, numerically using Boltzmann's method.
The porous medium is created using square barriers, with a regular arrangement that allows for the examination of cavity-scale complex flows. In the absence of a porous medium, the results are consistent with the corresponding analytical solutions. The results obtained in this case indicate that the flow and temperature distribution under the influence of the porous medium has changed and the existence of fixed obstacles in the computational range as a porous medium increases the thermal performance and the average Nusselt number.

Zehforoosh and Hosseinpoor [36] examined the natural displacement of a water-based nanofluid inside the package, partly filled with porous material with internal heat production. Maxwell and Brinkman models have been used to determine the solvent properties. The geometry examined in this study is shown in figure 6 the results show that increasing the nanosilver volume fraction will increase the Nusselt number in all porosities, which will increase in lower porosity. It also shows that changes in the porosity matrix conductivity ratio lead to two different modes of displacement and conduction within this environment. By increasing and decreasing the porosity, respectively, the displacement and guidance are amplified, which results in a minimum point in the porosity between 0.4 and 0.6 depending on the other parameters. The change in the high porosity conductivity ratio does not have an effect on the Nusselt number, but the increase in the conductivity ratio in the low porosity can increase the Nusselt number by as much as twice. Also, with Riley's increase in high porosity, penetration into the porous matrix increases, and in porosities below the outer wall of the porous matrix, the matrix is cooled, in both cases with the cooling of the matrix, the Nusselt number is greater in the entire domain Porosity is obtained. It was also concluded that using the lower porosity in the lower rillies and high porosity in the upper Riley, we can obtain the best Nusselt number factor. Nazari et al. [37] calculated the analytic and numerical value of the flow infiltration in a porous medium. In this research, a complete review of the modeling and calculating of the permeability coefficient in porous materials was first presented and then the porosity coefficient of a porous material with square geometry was calculated analytically and numerically. Selected geometry is for modeling a porous medium with a square section whose axis of fibers is vertically oriented to the flow. 


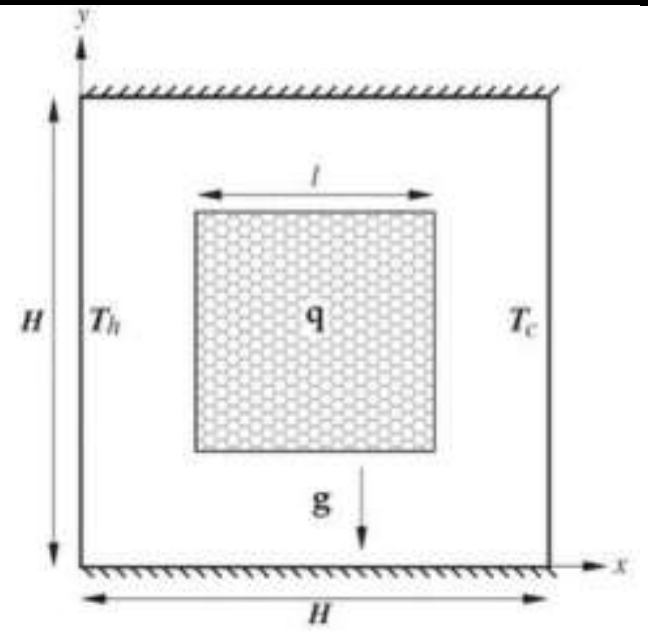

Fig. 6: Geometry examined by Zehforoosh and Hosseinpoor [36]

The governing flow equations are solved analytically after proper simplifications, and the explicit function for the coefficient of permeability is numerically analyzed. The predictions of numerical methods, analytical and analytical methods were compared with each other for the estimation of permeability of porous media with structural microfiber with square cross section. The results show that these methods are suitable for different porosities. Due to the Darcy equation in Reynolds number very low $\mathrm{Re}<<1$ (called creeping current) decreases the amount of pressure drop by increasing the permeability. Nebbali and Bouhadef [38] investigated the effect of using porous barriers on increasing the heat transfer of non-nuclide fluids in horizontal channels. In their work, they used the Brinkman-Forchimer model, which extended the Darcy model for porous media, and used two geometric models for the porous medium. The first model of the channel is with a porous barrier and the second model consists of two porous barriers alternating up and down the channel. In the work of Nabali and Bohadef the effect of various parameters such as Darcy number, Reynolds number, power law index and thermal conductivity ratio on heat transfer and flow pattern were investigated. They observed that quasi-plastic fluids produce the highest heat transfer with the least pressure drop in both modes. The results show that it is more beneficial from the thermal point of the first state, but dynamically, the second state is better; also other interesting results were obtained, such that the thermal efficiency of the second model can be increased through the paths. Madera et al. [39] solved the equations for effective environments for momentum modeling and heat transfer in a channel with parallel plates that were summited with porous material. To solve the boundary conditions at the boundary of the porous fluid, they solved the equations including location-dependent coefficients and solved the momentum transfer problem by the implicit integral equation based on the Green's function. The simulations were carried out in terms of porosity, plaster number, porosity size and thermal conductivity ratio, and similar to the previous work, it was determined that the increase in the heat transfer was increased by increasing the size of the porous material or the amount of mixing inside the canal. Chen Yang et al. [40] investigated the performance of the forced heat transfer heat transfer in a semi-permeable channel with porous material. In the study, the porous material was once in the center of the tube and once on the wall. They performed thermal equilibrium and heat imbalance analyzes in both cases in order to ascertain the accuracy of the assumption of thermal equilibrium in these two cases. Finally, it was concluded that the assumption of thermal imbalance for porous matter on the wall is necessary and the assumption of the thermal equilibrium for the porous material in the center is appropriate. It was also found that in the low range of pumping power, the heat transfer function of the pipe with the porous material in the center is better than the porous material in the wall, whereas in the pump power above it is the opposite.

Various arrangements of the porous medium inside the tube or the canal have been investigated so far. Huang and Vafai [41] investigated the effects of the simultaneous use of intermittent porous barriers and cavities on controlling heat transfer and surface friction on the outer surface, and showed that the formulation of this problem leads to the conclusion that the flow and transfer of heat to Seven parameters are dependent on the following: Reynolds number, Darcy number, Prantel number, Inertia parameter, Geometric dimensional parameters and number of barriers and porous cavities. They performed a deep analysis of the interaction between the flow of the cavities and the external flow. They also showed that changing some of the parameters could have significant effects on the characteristics of the outer momentum and the thermal boundary layer. Nield et al. [42] examined the condition of thermal imbalance in the fluid in the channel filled with porous media. They noted that the boundary condition of the fixed wall temperature had less effect on the thermal imbalance than other conditions, such as the condition of constant flux in the wall. They also concluded that the Nusselt number is highly dependent on the Pecklet number and the ratio of the effective thermal conductivity coefficient of the solid to the fluid, and has less dependence on the shape parameter and the Darcy number. In their work, they increased the ratio of effective thermal conductivity of the porous medium to the fluid to 10. In 1999, in the context of the use of porous materials in a double-walled heat exchanger, Alkam and Al-Namir, they investigated the heat transfer 
in the heat exchanger of two flow tubes that are in line with the porous medium [43]. The porous medium is placed on both sides of the inner tube and the flow of fluid is considered to be gentle. The results of the study show that heat transfer improves in the presence of porous matter. The heat exchanger efficiency also shows a significant increase in the heat capacity ratio. In 2001 [44], the researchers investigated the heat transfer of the plate heat exchanger in the central arrangement between the plates and showed that the presence of porous material in the central makeup reduced the thickness of the boundary layer and consequently Increases heat transfer. Poulikakos and Kazmierczak's research [45] on compulsory displacement in a channel, which is partially occupied by porous material. The porous material is clinging to the channel wall. They have solved the problem for two situations between two parallel planes and a channel with a circular cross-section. Also, in his results, the effects of a number of parameters such as the thickness of porous area adhering to the wall, the Darcy number and the effective thermal conductivity coefficient of the porous region to the thermal conductivity of the fluid are investigated on the fluid flow and heat transfer in the channel. The effect of the above parameters on the flow characteristics of the fluid and the heat transfer were qualitatively investigated for the two geometries, for the boundary conditions, the temperature and constant thermal flux in the wall were similar. The other result is the linear dependence of the Nusselt number on the thickness of the porous region. In other words, there is a critical thickness for the porous region in which the Nusselt number reaches a minimal value. Xu et al. [46] in an analytic study examined the forced flow inside the filled pipe. The solution flow in this study was fully developed and assisted by a two-equation model, using the Brinkman model for flow analysis. The assumption of heat transfer between two fluid and solid phases is a local thermal imbalance and has been used to solve equations in the case of two fluid and solid phase assumptions. In this study, the temperature and velocity profiles are implicit equations for the coefficient of friction and Nusselt number. The effect of other variables on heat transfer has also been studied. Nimvari et al. [47] studied the flow and heat transfer in porosity channel channels in detail. In this study, where two layers were considered, the porous material was clamped to the surface of the tube in a state, and in the second case it was placed in the center of the tube. For both layers of different thicknesses and different Darcy numbers have been investigated. For placement of the fastening to the wall, the Nusselt number in the thicknesses between 0.6 and 0.7 for the minimum number of Darcy has been found to be minimal, also for laying in the center of the channel, the maximum Nsult number in the thicknesses of 0.83, 0 and 0.9 in Darcy numbers are $0.01,0.001$, and $0001 / 0$. They concluded that as the amount of the Darcy number decreases, the thickness in which the minimum number of Nusselt numbers is decreasing. Aguilar-Madera et al. [48] investigated the effective equations of heat transfer and momentum in the channel filled with porous medium. The equations are solved using a location-dependent coefficient to avoid assuming certain conditions in the boundaries of fluid collision with the porous medium. Also, energy equations are solved by numerical solution using finite element method. This numerical simulation has been performed based on a number of data, such as porosity coefficient and Pecklet number. Rochette and Clain [49], in a study using a new microscopic model, sought to evaluate the local thermal heat load assumption in the solid phase of porous matter. To do this, they simulated the gas flow inside the porous material using the Euler model. In the geometry examined, the porous material completely fills inside the tube. They first used the classical method of local thermal imbalance, then introduced a new model in which the porosity depth is used.

Peng et al. [50] studied numerical parameters affecting the porous medium and its effect on the coefficient of performance improvement and heat transfer. In numerical simulation, they examined the thermal conductivity of the porous material for the porous material at the center of the tube. The flow inside the tube was calm and fully developed. The porosity of the porous medium was 0.9 and the Reynolds number range was 25-2000. Also, the thermal conductivity of the porous material varied from 0.1 to $200 \mathrm{~W} / \mathrm{m}$.C. This simulation was carried out in four different modes: in the two fluid states of the air and in a fluid state of water, in the fourth state the fluid had a density of one fourth of the water, while the other properties were all similar to water. According to the results of this simulation, Nusselt number and coefficient of improvement have a uniform increase in yield with porosity conductivity coefficient and Reynolds number.

\section{EXPERIMENTAL STUDIES}

Pavel and Mohamad [51] have investigated the effects of insertion of metal porous material in the form of a grid in the center of the pipe, on numerically and experimentally examining the heat transfer of a pipe that is subject to constant and uniform flux. They investigated the porosity, porous diameter and conduction heat transfer coefficient plus the Reynolds number. Results for Reynolds numbers ranging from 1,000 to 4,500 including both relaxed and turbulent regimes. The results showed that this placement, which in the case where the diameter of the porous material is close to the diameter of the pipe, increases the 
heat transfer rate by the cost of the logical pressure drop. The maximum increase in the mean value of the Nusselt number is reported to be 2.5 times as much as the nonporous medium. This condition is achieved when the entire tube is filled with porous medium and has porosity equal to $98.1 \%$ and a Reynolds number of 4500 . The porous material used in this experiment was aluminum commercial lace $\left(\mathrm{k}=177 \mathrm{w} / \mathrm{m}^{\wedge} 2 . \mathrm{k}\right)$. Nazari et al. [52] investigated the experimental forced displacement of nanofluids in a horizontal tube containing porous material. In this experiment, they placed a tube containing porous material under constant temperature conditions and carried out 700 to 5000 experiments in the range of Reynolds numbers. The results show that the addition of nano powder to base fluid leads to a significant increase in heat transfer in the tube. It was also concluded that the tube containing porous material with a porosity of $50 \%$ and $63 \%$ would increase the heat transfer significantly (about twice) compared with the empty tube. In another experimental study, Nazari et al. [53] performed a test for forced heat transfer in a cylindrical duct channel with internal heat production. Dry air has been used as an agent fluid in the process of cooled hot spherical beads. The internal temperature with electromagnetic induction heating method is uniformly produced in the spherical metal particles used in the test. The study is in constant flow mode and in the turbulent flow regime in the range of 4500 to 9500 Reynolds number. Various parameters due to changes in grain diameter, fluid velocity and amount of heat generated on forced transfer heat transfer in porous channel have been studied. The results of the present work indicate an increase in the forced heat transfer coefficient of the air in the porous channel by increasing the Reynolds number, as well as the porous channel of the pressure drop. On the other hand, by reducing the diameter of the grains, the forced heat transfer and the pressure drop in the canal have increased and the fluid temperature has decreased. Compared to the work done with water, the forced heat transfer coefficient for air fluid is lower and the pressure drop is lower than the water fluid. Angirasa [54] performed experiments to increase the amount of heat transfer by placing metal fibrous materials with two porosities of 97 and 93 percent in the canal. The output of Reynolds number changes from 17000 to 29000 and the input power of the pump is $7 / 3$ and 9/2 watts. An increase of 3 to 6 times the Nusselt number compared to a non-porous channel has been reported. Hetsroni et al. [55] investigated the heat transfer and pressure drop in a rectangular channel with a porous material in the bed by laboratory method. In this experiment, low porosity was investigated for heat dissipation in small-sized devices with high power, and the results showed that the wells would be very efficient, although the heat transfer increased as the heat pump increased. Pamuk, and Ozdemir [56] experimentally investigated the heat transfer in a porous medium under fluctuating water flow. A total of 54 experiments performed with frequency, fluid displacement length and different incoming heat. They used two steel bullet models to form a porous medium in the tube. They provided a model for calculating Nusselt numbers in their work. Wang and Guo [57] investigated numerically and experimentally the effect of inserting metal fibers into a square channel and without contact with the channel wall. The results showed that the temperature profile of the fluid passing through the fibers would be more uniform and the temperature of the liquid near the wall would be more severe, thereby increasing the heat transfer. Their geometry is shown in figure 7.

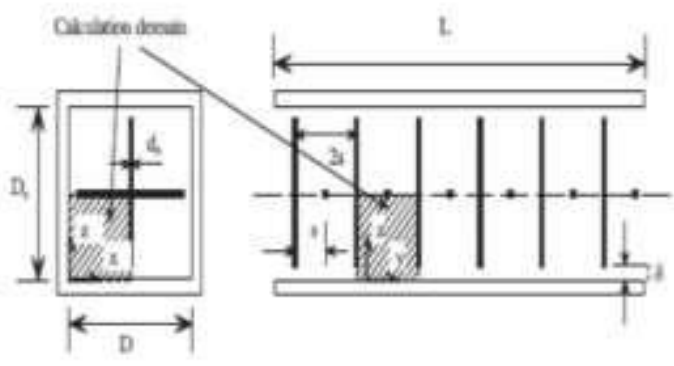

Fig. 7: Schematic research of Wang and Guo [57]

Huang et al. [58] examined the increase in heat transfer by embedding the porous medium at the center of a tube numerically and experimentally. In their work, they used diameters of copper with different diameters, which were inserted at different intervals, and used the boundary condition of constant flux. They used air as a fluid in a wide range of Reynolds number $(1000<\operatorname{Re}<19000)$. It also used three different porosity of $0.951,0.966$, and 0.975 in experiments. The effect of radius on the function of the system was numerically investigated. Experimental and numerical results showed that the transfer heat transfer substantially increased in porous medium placements close to the pipe diameter, and the flow resistance also increased at a reasonable rate, especially in a slow flow. In sum, they introduced this method, an effective solution to increase heat transfer. Jiang et al. [59] investigated the empirical and numerical research of forced heat transfer in a canal filled with porous media. The schematic geometry used is shown in figure. 8. They studied the effects of temperature distribution, variable properties due to pressure variation, porosity particle diameter, thermal conductivity of particles and fluid velocity. The experimental and numerical results obtained for the coefficient of friction in the porous medium were in good agreement with the value obtained from the formula. The porous medium also significantly increased 
the pressure drop across the canal, compared with the empty channel. Heat transfer has decreased with decreasing the diameter of the porous particles and increased with increasing the conductivity of the particles.

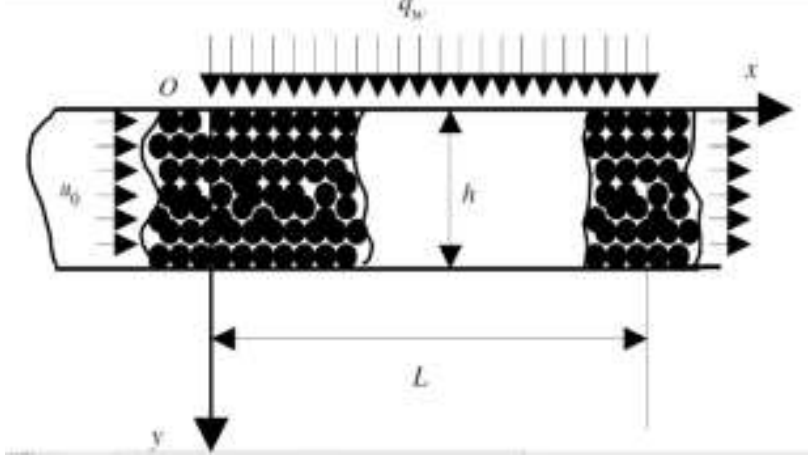

Fig. 8: Geometry examined by Jiang et al. [59]

Sarada et al. [60] investigated numerically and experimentally the effect of mesh insertion in a horizontal tube on increasing the heat transfer of the flow of turbulent fluid by air. They observed the most Nusselt number, equal to 2.51 times the simple tube, at a mesh radius of 0.8 and a distance of $50 \mathrm{~mm}$. Also, the highest drop in pressure drop that is 1.23 times of the simple tube was in the mesh radius of 0.945 .

\section{CONCLUSION}

Placement of porous materials in the fluid path is one of the passive ways of increasing heat transfer in heat exchangers. In this paper, recent studies on the effect of using porous media on increasing the amount of heat transfer in heat exchangers by applying porous environments with porosity percentage, gender and geometric structure in the flow path, using numerical simulations and studies Laboratory was studied. However, each of the results is related to the particular case and its conditions, but studies have shown that the presence of porous media in the flow path improves the thermal conductivity matrix and effective heat capacity of the flow. A porous solid-state environment also increases the heat transfer velocity especially in systems where gas is flowing. It seems that, given the increasing human need for energy and the needs to prevent its loss, many researchers are working to increase the efficiency and efficiency of energy production or conversion systems using porous media.

\section{REFERENCES}

[1] S. Whitaker, Introduction to Fluid Mechanics, New Jersey: Prentice-Hall, 1968.

[2] G. F. Hewitt, G. L. Shires, T. R. Bott, Process Heat Transfer, Second Edittion., New York: CRC Press, 1994.
[3] A. E. Bergles, Heat transfer enhancement, the encouragement and accommodation of high heat fluxes, J. Heat Transfer, vol. 119, Issue 1, pp. 8-19, 1997.

[4] A. E. Bergles, R. L. Webb, Augmentation of Convective Heat and Mass Transfer, New York: American Society of Mechanical Engineers, 1970.

[5] D. A. Nield, A. Bejan, Convection in porous media, fourth Edittion, New York: Springer, 2013.

[6] J. G. Fourie, J. P. Du Plessis, Pressure drop modelling in cellular metallic foams, Chemical Engineering Science, vol. 57, No. 14, pp. 27812789, 2002.

[7] B. Nie, X. Liu, L. Yang, J. Meng, X. Li, Pore structure characterization of different rank coals using gas adsorption and scanning electron microscopy, Fuel, vol. 158, pp. 908-917, 2015.

[8] J. Bear, Dynamics of fluids in porous media, New York: Courier Corporation, 2013.

[9] M. Kaviany, Principles of heat transfer in porous media, New York: Springer, 1995.

[10]D. A. Nield, A. Bejan, Convection in Porous Media, fourth Edition, New York: Springer, 2013.

[11]A. E. Scheidegger, The Physics of Flow through Porous Media, Third Edition, Toronto: University of Toronto Press, 1974.

[12] A. Bejan, J. L. Lage, Convective Heat and Mass Transfer in Porous Media, pp. 823-845, Netherlands: Springer, 1991.

[13]R. Webb, E. Eckert, Application of rough surfaces to heat exchanger design, International Journal of Heat and Mass Transfer, Vol. 15, No. 9, pp. 16471658, 1972.

[14]D. A. De Vries, The theory of heat and moisture transfer in porous media revisited, International Journal of Heat and Mass Transfer, vol. 30, Issue 7, pp. 1343-1350, 1987.

[15]C. L. Tien, K. Vafai, Convective and radiative heat transfer in porous media, Advances in Applied Mechanics, vol. 27, pp. 225-281, 1989.

[16] R. Webb, Performance evaluation criteria for use of enhanced heat transfer surfaces in heat exchanger design, International Journal of Heat and Mass Transfer, Vol. 24, No. 4, pp. 715-726, 1981.

[17] R. Webb, E. Eckert, Application of rough surfaces to heat exchanger design, International Journal of Heat and Mass Transfer, Vol. 15, No. 9, pp. 16471658, 1972.

[18] Whitaker, S. "Flow in porous media I: A theoretical derivation of Darcy's law, Transport in porous media, vol. 1, Issue 1, pp. 3-25, 1986.

[19]S. Ergun, Fluid flow through packed columns, Chem. Eng. Prog, Vol. 48, No. 2, pp. 89-94, 1952. 
[20] R. L. Webb, Principle of Enhanced Heat Transfer, second Edition, New York: CRC Press, 2005.

[21] G. Lauriat, G. Riad, Forced convective heat transfer in porous media, New York: Dekker, 2000.

[22] A. A. Mohamad, Heat transfer enhancements in heat exchangers fitted with porous media. Part 1: Constant wall temperature, International journal of Thermal Sciences, vol. 42, Issue 4, pp. 385-395, 2003.

[23] M. Siavashi, H. R. Talesh-Bahrami, H. Saffari, Numerical investigation of flow characteristics, heat transfer and entropy generation of nano-fluid flow inside an annular pipe partially or completely filled with porous media using two phases mixture model, Energy, vol. 93, Part 2, pp. 2451-2466, 2015.

[24]H. Shokouhmand, F. Jam, M. R. Salimpour, Simulation of laminar flow and convective heat transfer in conduits filled with porous media using Lattice Boltzmann Method, International Communications in Heat and Mass Transfer, vol. 36, Issue 4, pp. 378-384, 2009.

[25] M. Nazari, M. M. Shahmardan, S. Mosayebidorcheh, Analytical Solution of Steady Heat Transfer in a Multi-Layer Porous Media under Local Thermal Non-Equilibrium Condition, Modares Mechanical Engineering, vol. 12, Issue 4, pp. 1-8, 2012. (in Persian)

[26] M. Maerefat, A. Jamarani, M. Eshagh-Nimvari, Numerical study of heat transfer in double-tube heat exchanger filled with porous material in a turbulent fluid flow, Modares Mechanical Engineering, vol. 16, Issue 3, pp. 173-184, 2016. (in Persian)

[27] M. Maerefat, A. Jamarani, M. Eshagh-Nimvari, Introducing proper definition of the Nusselt number for fluid flow in pipe partially filled with porous media, Modares Mechanical Engineering, vol. 15, Issue 6, pp. 278-286, 2015. (in Persian)

[28] M. Maerefat, A. Jamarani, M. Eshagh-Nimvari, Numerical investigation of nanofluid's effect on heat transfer in a pipe partially filled with porous material in a turbulent fluid flow, Modares Mechanical Engineering, vol. 16, Issue 6, pp. 255-258, 2016. (in Persian)

[29] H. Hamidi-Moghadam, M. Daie, H, Amiri, H. Erfan-Niya, Investigating the effects of viscous dissipation on compulsory displacement inside the porous cylindrical channel and solving the governing equations, Accessed on 17 April 2014; https://www.civilica.com/Paper-TCPCO01TCPCO01_234.html. (in Persian)

[30] H. Shokouhmand, A. Ejlali, Study of heat transfer in an array of two-dimensional porous fins, Journal of the College of Engineering, vol. 39, Issue 1, pp. 3140, 2005. (in Persian)

[31]M. R. Rezaie, M. J. Maghrebi, Numerical investigation of conjugate natural convection heat transfer in porous enclosure with lattice Boltzmann method, Journal of Solid and Fluid Mechanics, vol. 5, Issue 2, pp. 217-231, 2015. (in Persian)

[32] M. Nazari, E. Shakeri-Nejad, M. H. Kayhani, Natural Convection in a Porous Medium with Heat Generation: Self-Similar Solution, Journal of Applied and Computational Sciences in Mechanics, vol. 24, No. 2, pp. 45-56, 2013. (in Persian)

[33] M. Nazari, E. Shakeri-Nejad, M. H. Kayhani, Heat and mass transfer in a porous enclosure in the presence of biochemical heat source, Modares Mechanical Engineering, vol. 12, Issue 2, pp. 144152, 2012. (in Persian)

[34] M. Taeibi-Rahni, M. Khayat, R. Aliabbasi, Numerical Simulation of Forced Convection Flow through a Flattened Pipe Using Porous Material in the Central and Boundary Arrangement, Aerospace Mechanics Journal, vol. 9, No. 1, pp. 77-85, 2013. (in Persian)

[35] M. M. Kayhani, R. Mohebi, Numerical Investigation of Fluid Flow and Heat Transfer on the Porous Media Between Two Parallel Plates Using the Lattice Boltzmann Method, , Aerospace Mechanics Journal, vol. 9, No. 1, pp. 63-76, 2013. (in Persian)

[36] A. Zehforoosh, S. Hossainpour, Porous Matrix properties effect on the internal heat generation in a square cavity saturated with $\mathrm{Cu}$-Water nanofluid, Modares Mechanical Engineering, vol. 14, Issue 16, pp. 34-44, 2015. (in Persian)

[37] M. Nazari, A. R. Salehi, M. Khaksar, Analytical and numerical calculation of flow permeability in a porous medium with square cross section, Modares Mechanical Engineering, vol. 12, Issue 1, pp. 21-32, 2012. (in Persian)

[38] R. Nebbali, K. Bouhadef, Non-Newtonian fluid flow in plane channels: Heat transfer enhancement using porous blocks, International Journal of Thermal Sciences, Vol. 50, Issue 10, pp. 1984-1995, 2011.

[39] C. G. A. Madera, F. J. V. Parada, B. Goyeau, J. A. O. Tapia, Convective heat transfer in a channel partially filled with a porous medium, International Journal of Thermal Sciences, Vol. 50, Issue 8, pp. 1355-1368, 2011.

[40]C. Yang, A. Nakayama, W. Liu, Heat transfer performance assessment for forced convection in a tube partially filled with a porous medium, International Journal of Thermal Sciences, Vol. 54, pp. 98-108, 2012. 
[41] P. C. Huang, K. Vafai, Passive alteration and control of convective heat transfer utilizing alternate porous cavity-block wafers, International Journal of Heat and Fluid Flow, Vol. 15, Issue 1, pp. 48-61, 1994.

[42] D. A. Nield, A. V. Kuzentsov, M. Xiong, Effect of local thermal non-equilibrium on thermally developing forced convection in a porous media, International Journal of Heat and Mass Transfer, Vol. 45, Issue 25, pp. 4949-4955, 2002.

[43] M. Alkam, M. Al-Nimr, Improving the performance of double-pipe heat exchangers by using porous substrates, International Journal of Heat and Mass Transfer, Vol. 42, No. 19, pp. 3609-3618, 1999.

[44] M. Alkam, M. Al-Nimr, M. Hamdan, Enhancing heat transfer in parallelplate channels by using porous inserts, International Journal of Heat and Mass Transfer, Vol. 44, No. 5, pp. 931-938, 2001.

[45]D. Poulikakos, M. Kazmierczak, Forced Convection in a Duct Partially Filled with a Porous Material, Trans. ASME J. Heat Transfer, Vol. 109, Issue 3, pp. 653-662, 1987.

[46] H. J. Xu, Z. G. Qu, W. Q. Tao, Analytical solution of forced convective heat transfer in tubes partially filled with metallic foam using the two-equation model, International Journal of Heat and Mass Transfer, vol. 54, Issue 17-18, pp. 3846-3855, 2011.

[47] M. E. Nimvari, M. Maerefat, M. H. El-Hossaini, Numerical simulation of turbulent flow and heat transfer in a channel partially filled with a porous media, International journal of thermal sciences, vol. 60, pp. 131-141, 2012.

[48] G. C. Aguilar-Madera, F. G. Valdes-Parada, B. Goyeau, J. A. Ochoa-Tapia, Convective heat transfer in a channel partially filled with a porous medium, International Journal of Thermal Sciences, vol. 50, Issue 8, pp. 1355-1368, 2011.

[49]D. Rochette, S. Clain, Local heat transfer of compressible fluid in porous media: application to the HBC fuse, International journal of heat and fluid flow, vol. 26, Issue 2, pp: 322-333, 2005.

[50] C. Peng, T. Ming, Y. Tao, Thermal and hydraulic performances of a tube filled with various thermal conductivities of porous media, International Journal of Heat and Mass Transfer, vol. 81, pp. 784-796, 2015.

[51]B. I. Pavel, A. A. Mohamad, An experimental and numerical study on heat transfer enhancement for gas heat exchangers fitted with porous media, International Journal of Heat and Mass Transfer, Vol. 47, Issue 23, pp. 4939-4952, 2004.

[52] M. Nazari, M. Ashouri, M. H. Kayhani, Experimental investigation of forced convection of nanofluids in a horizontal tube filled with porous medium, Modares Mechanical Engineering, vol. 14, Issue 7, pp. 109-116, 2014. (in Persian)

[53]M. Nazari, D. Jalili-Vahid, S. Khanjari, Experimental investigation of force convection heat transfer in porous channel with internal heat generation, Modares Mechanical Engineering, vol. 15, Issue 1, pp. 203-210, 2015. (in Persian)

[54]D. Angirasa, Experimental investigation of forced convection heat transfer augmentation with metallic fibrous materials, Int. J. Heat Mass Transfer, vol. 45, No. 4, pp. 919-922, 2002.

[55]G. Hetsroni, M. Gurevich, R. Rozenblit, Sintered porous medium heat sink for cooling of high-power mini-devices, International Journal of Heat and Fluid Flow, Vol. 27, Issue 2, pp. 259-266, 2006.

[56] M. T. Pamuk, M. Ozdemir, Heat transfer in porous media of steel balls under oscillating flow, Experimental Thermal and Fluid Science, Vol. 42, pp. 79-92, 2012.

[57]S. Wang, Z. Y. Guo, Z. X. Li, Heat transfer enhancements by using metallic filament insert in channel flow, International Journal of Heat and Mass Transfer, Vol. 44, Issue 7, pp. 1373-1378, 2001.

[58]Z. F. Huang, A. Nakayama, K. Yang, C. Yang, W. Liu, Enhancing heat transfer in the core flow by using porous medium insert in a tube, International Journal of Heat and Mass Transfer, Vol. 53, Issue 5-6, pp. 1164-1174, 2010.

[59] P. X. Jiang, G. S. Si, M. Li, Z. P. Ren, Experimental and numerical investigation of forced convection heat transfer of air in non-sintered porous media, Experimental Thermal and Fluid Science, vol. 28, Issue 6, pp. 545-555, 2004.

[60] S. N. Sarada, A. V. S. R. Raju, K. K. Radha, Experimental and Numerical Analysis of Turbulent Flow Heat Transfer Enhancement in a Horizontal Circular Tube Using Mesh Inserts, Journal of Energy and Power Engineering, Vol. 4, No. 7, pp. 32-41, 2010. 